\title{
Ulnar Collateral Ligament Reconstruction: Allograft Versus Autograft - A Systematic Review
}

ISSN: 2576-8875

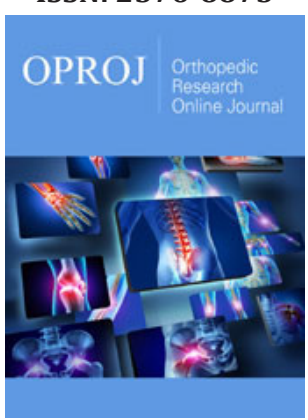

*Corresponding author: Amy Singleton BS, Department of Orthopedics, Mercy Health St Vincent Medical Center, 2409 Cherry Street, USA

Submission: 漈 August 16, 2021

Published: 沮August 31, 2021

Volume 8 - Issue 4

How to cite this article: Austin Moore DO, Dylan Mathews DO, Julie Stausmire, Amy Singleton BS, Kirk Davis DO, Anil Gupta MD. Ulnar Collateral Ligament Reconstruction: Allograft Versus Autograft - A Systematic Review. Ortho Res Online J. 8(4). OPROJ. 000694. 2021.

DOI: $10.31031 /$ OPROJ.2021.08.000694

Copyright@: Amy Singleton BS, This article is distributed under the terms of the Creative Commons Attribution 4.0 International License, which permits unrestricted use and redistribution provided that the original author and source are credited.

\author{
Austin Moore D0 ${ }^{1}$, Dylan Mathews D0 ${ }^{1}$, Julie Stausmire ${ }^{2}$, Amy Singleton BS ${ }^{*}$, \\ Kirk Davis $\mathrm{DO}^{1}$ and Anil Gupta $\mathrm{MD}^{3}$ \\ ${ }^{1}$ Department of Orthopedics, Mercy Health St Vincent Medical Center, 2409 Cherry Street, USA \\ ${ }^{2}$ Regional Academic Affairs, Mercy Health St Vincent Medical Center, 2215 Cheery Street, USA \\ ${ }^{3}$ Toledo Orthopedic Surgeons, 2865 N Reynolds Rd, USA
}

\begin{abstract}
Background: Ulnar Collateral Ligament (UCL) reconstruction of the elbow, a Tommy John surgery, is a common procedure often performed in throwing athletes. Autograft tendon has traditionally been utilized, although recently allograft tendon has been of interest due to the possibility of decreasing donor site morbidity. There is a lack of literature on patient outcomes and complication rates following the use of allograft tendon for UCL reconstruction. Currently, there is no consensus on utilizing autograft versus allograft tendon for UCL reconstruction.
\end{abstract}

Methods: A PRISMA compliant literature search in online databases Medline, Cochrane and Embase was performed for level 4 and higher studies through June 2019. Any studies reporting clinical outcome results of allograft reconstruction were included. Exclusion criteria included studies unrelated to primary UCL reconstruction, studies specifically looking at revision UCL reconstruction, epidemiological studies, case reports, studies lacking raw data, technique articles, biomechanical studies, cadaveric studies, studies with outcomes unrelated to graft choice, and studies with outcomes unrelated to the use of allograft tendon for reconstruction. Studies were analyzed for graft type, functional scores, return to play rate, and complication rates.

Results: Three out of 103 studies met inclusion criteria. Two cohort studies and 1 retrospective review were included in this review. No significant differences in functional outcomes regardless of scoring system utilized, return to play rate, or complication rates were found between allograft versus autograft.

Conclusion: The use of allograft tendon appears to have similar outcomes regarding functional scores, return to play, and complication rates compared to autograft tendon. Use of allograft tendon seems to be a viable option for UCL reconstruction, though further studies are needed.

Keywords: Allografts; Autografts; Elbow; Ulnar collateral ligament reconstruction; Tommy john; Elbow instability

Abbreviations: UCL: Ulnar Collateral Ligament; PRISMA: Preferred Reporting Items for Systematic Reviews and Meta-Analyses; KJOC: Kerlan-Jobe Orthopaedic Clinic Shoulder and Elbow Scores; MEPS: Mayo Elbow Performance Scores; OES: Oxford Elbow Scores; DASH: Disabilities of the Arm, Shoulder and Hand Scores

\section{Introduction}

Injury to the medial Ulnar Collateral Ligament (UCL) was originally described by Waris [1] in 1946 in Javelin throwers. Today, baseball pitchers are an increasingly common demographic who sustain injuries to the UCL [2]. Commonly, UCL injuries in pitchers are overuse injuries due to the repetitive valgus force applied during the acceleration phase of throwing [2]. Throwing athletes with acute UCL injuries may describe a pop associated with pain and difficulty with throwing. They often have pain during the late cocking and early acceleration phases of throwing. This leads to decreased velocity and accuracy with throwing [3]. UCL injuries are not only limited to the throwing athletes; injury can also occur during any activities that cause repetitive valgus moments on the elbow. Traumatic elbow dislocation can 
also lead to acute UCL injury. On physical exam, the most common finding is tenderness to palpation over the medial elbow at the UCL origin. Patients may or may not report having pain at rest, and many chronic tears are symptomatic only during a throwing motion [2].

The medial UCL is the primary restraint to valgus stress on the elbow, and anatomically is divided into 3 components, the anterior bundle, posterior oblique ligament, and transverse ligament [4]. In the anterior bundle, the anterior oblique ligament originates at the anteroinferior ridge of the medial epicondyle to the sublime tubercle, and along its course subdivides into anterior and posterior bands of which the anterior band is the primary valgus restraint [5]. The anterior band of the anterior bundle is the primarily stabilizer from 30 to 120 degrees of flexion while the posterior band has the same function at terminal phase of elbow flexion [6]. The transverse ligament does not contribute to stability of the elbow. During the late cocking and acceleration phases of throwing, a significant valgus stress is placed on the elbow, which leads to repetitive micro-trauma to the anterior band of the UCL, which can eventually lead to rupture.
In 1974, Jobe et al. [7] performed the first successful medial ulnar collateral ligament reconstruction on baseball pitcher Tommy John, leading to the procedure's moniker. Later on, in 1986, Jobe et al. [7] described a technique for reconstruction of the ligament with use of ipsilateral palmaris longus tendon autograft via flexor pronator mass detachment with transposition of ulnar nerve. Since then, there have been several modifications and variations of Dr. Jobe's original technique. There have also been an increasing number of tendon graft origins for UCL reconstruction that have been utilized including palmaris, hamstring, plantaris, and Achilles autograft. Unfortunately, with autograft harvest, there can be complications involving the graft site including graft site pain, infection, numbness, and even incidental harvesting of the median nerve. With recent improved tissue processing techniques, allograft has become a viable treatment option to avoid donor site morbidity.

To date, there has been a paucity of literature surrounding outcomes following UCL reconstruction with allograft. The purpose of this study is to evaluate outcomes after UCL reconstruction with allograft versus with autograft tendons in regard to functional scores, return to play rate, and complication rates.
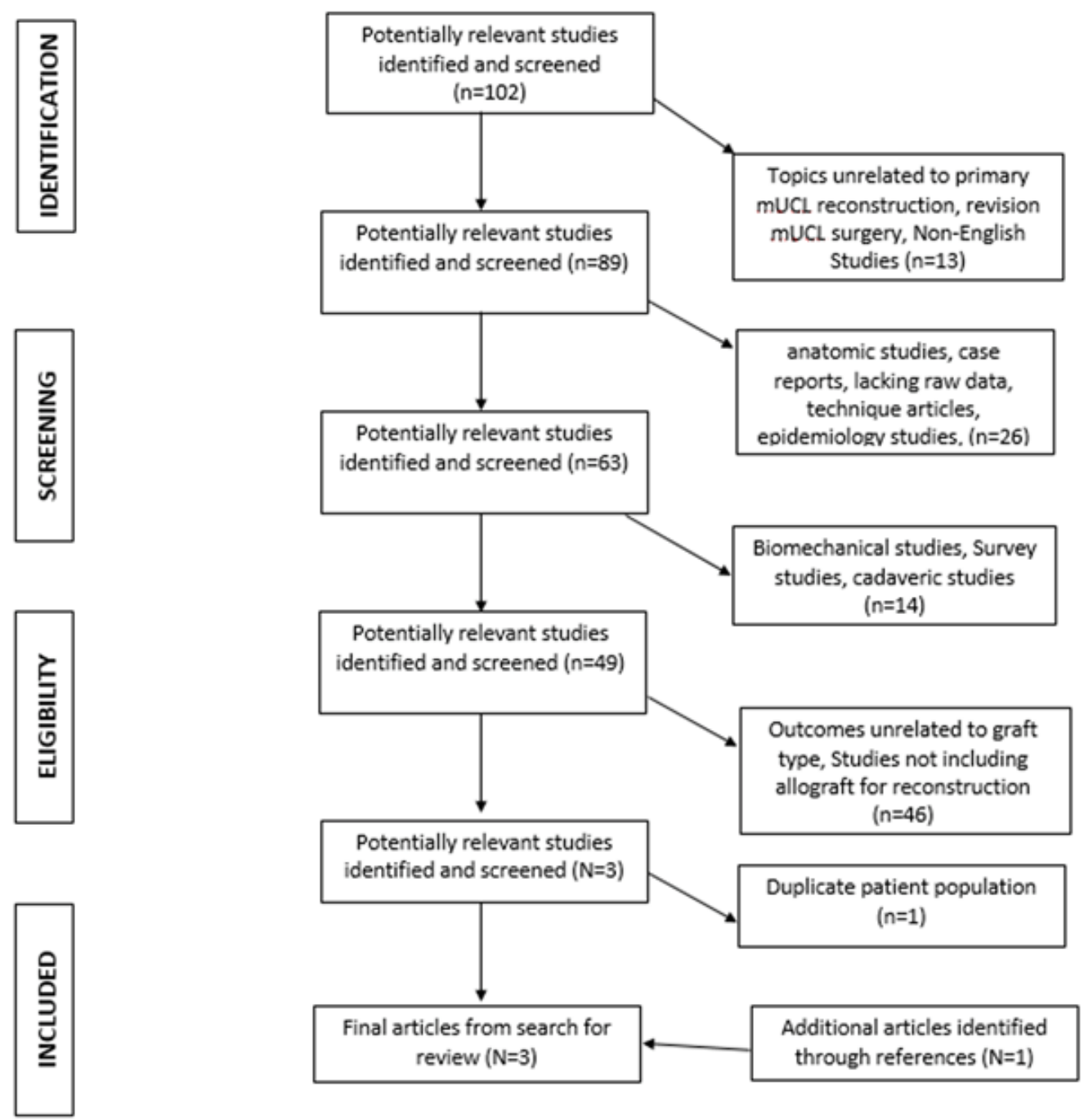

Figure 1: PRISMA Flowsheet for study inclusion. 


\section{Methods}

A systematic review was first registered on Prospero and a literature search was performed using Preferred Reporting Items for Systematic Reviews and Meta-Analyses (PRISMA) guidelines (Figure 1); [8,9]. The search was performed utilizing Medline, Cochrane, and Embase electronic databases, first in March and then in June 2019 to identify the most contemporary articles. Full text versions of articles were reviewed by two independent resident physician reviewers. Primary search terms included: ulnar collateral ligament reconstruction, allograft, autograft, Tommy John, reconstructive surgical procedures, and surgical repair used in combination and/or independently.

Inclusion criteria were defined as any studies reporting clinical outcome results of allograft reconstruction. Exclusion criteria for studies were studies in a non-English language, studies unrelated to primary medial UCL reconstruction, studies specifically looking at revision medial UCL reconstruction, epidemiological studies, case reports, studies lacking raw data, technique articles, biomechanical studies, cadaveric studies, studies with outcomes unrelated to graft choice, and studies with outcomes unrelated to the use of allograft tendon for reconstruction.

Studies with levels of evidence of 4 or higher were included and then reviewed for identical patient populations. Two studies by Erickson et alwere identified which included the same patient population $[10,11]$. The study with the higher level of evidence between these two studies was included in our review. The references of our included studies were then cross examined for any additional studies which could be included, resulting in one additional study. Studies identified were then reviewed for graft type used and various functional outcomes, return to play, and complications

\section{Results}

Out of 103 studies initially identified in our literature search, 3 met all inclusion criteria. Two studies were level 3 cohort studies, and one study was a level 4 retrospective review. Of the studies identified, no significant differences were found between functional outcome scores, return to play, or return to play at previous level of competition.

In Erickson et al. [11] a level 3 cohort study, 85 patients between 2004-2013 underwent UCL reconstruction. 11 patients underwent reconstruction with allograft tendon, 20 underwent reconstruction with hamstring autograft tendon, and 54 underwent reconstruction with palmaris autograft tendon. Functional outcomes scores were reported using the Kerlan-Jobe Orthopaedic Clinic Shoulder and Elbow Score (KJOC) and the Timmerman-Andrews Score. KJOC scores for palmaris autograft, hamstring autograft, and allograft were $91.67 \pm 8.59, \quad 89.62 \pm 9.12$, and $91.66 \pm 3.20$ respectively $(p=0.251)$. Timmerman-Andrews Score between the palmaris autograft, hamstring autograft, and allograft were 91.67 \pm 8.59 , $93.75 \pm 5.82$, and $94.55 \pm 4.72$ respectively $(p=0.181)$. Percentage of patients who returned to play at the same level or higher as preinjury was $92.59 \%$ for palmaris autograft, 95\% for hamstring autograft, and $100 \%$ for allograft reconstruction ( $\mathrm{p}=0.999)$. No difference in complications between the type of graft used was identified. Complications included reoperation for ulnar nerve transposition $(n=6)$, ulnar stress fracture $(n=1)$, loss of motion requiring reoperation with elbow arthroscopy $(n=1)$, and one UCL re-tear, ultimately treated non-operatively.

Merolla's et al. [12] study included 15 patients who underwent UCL reconstruction between 2006-2012. Of the 15 patients, 5 underwent reconstruction with palmaris autograft and 10 underwent reconstruction with semitendinosus allograft. Functional outcome scores were reported using the Mayo Elbow Performance Score (MEPS), Oxford Elbow Score (OES), and Disabilities of the Arm Shoulder and Hand score (DASH). Overall MEPS scores improved from 56.3 preoperatively to 93.8 postoperatively, OES from 23.9 to 45.8 , and DASH from 31.8 to 1.81 . This study reported improvement in post-operative functional scores in each scoring system, though there were no significant difference noted between any of the above outcome scores between autograft and allograft groups ( $p>0.05$ ). This study did not report complication rates, though they did identify calcific deposition in all grafts, 10 at the humeral insertion and 5 at the ulnar insertion. They did not report on return to play rates.

A retrospective study by Savoie et al. [8] evaluated functional outcome scores, return to play rate, range of motion, and complications at 2 years in 116 patients who underwent ulnar collateral ligament reconstruction with allograft tendon. Of the 116 patients, gracilis allograft was used in 100 patients, and semitendinosus allograft was used in 16 patients. Functional outcome scores were reported with the Conway-Jobe scale. 93 patients reported excellent outcomes (81\%), 15 patients reported good outcomes (13\%), 8 patients reported fair outcomes (7\%), and no patient reported poor outcomes. 33 patients $(28.4 \%)$ returned to play above preinjury level, $64(55.1 \%)$ returned at preinjury level, 13 (11.2\%) returned below preinjury level, and 6 (5.2\%) did not return to play, 3 of which were for reasons unrelated to their surgery. Average return to competition was 9.5 months. Postoperative complications, reported in 7 patients (6\%), included 1 ulnar neuropathy, 2 late sensory neuropathy, 2 postoperative wound issues, 1 medial humeral epicondyle fracture, and 1 flexorpronator muscle and tendon tear. No graft failures were reported. Savoie et al concluded that outcomes following reconstruction with allograft versus autograft were similar in regard to ConwayJobe outcome scores, return to play rate, range of motion, and complication rates.

\section{Discussion}

Ulnar collateral ligament tears occur most often in pitchers and other overhead throwing athletes resulting in instability of the elbow joint [10]. Reconstruction of the UCL with autograft tendon has been a mainstay in treatment since the late 1900's. Although successful as a treatment for returning elbow stability, the use of autograft also provides the potential opportunity for donor site morbidity as well as extended operating room time. Donor site morbidity ranges from minor symptoms such as donor site pain 
and numbness, to severe complications, including infection and damage to surrounding structures. Numbness in the infrapatellar branch of the saphenous nerve distribution has been reported in up to $88 \%$ of patients who underwent hamstring autograft harvest [13]. A more severe and detrimental complication of incidental harvest of the median nerve has also been reported with palmaris longus tendon autograft harvest [14]. The use of allograft tendon for UCL reconstruction appears to be an appropriate alternative for reducing these potential complications that can be seen with autograft harvesting.

Functional outcomes after UCL injury and surgical repair are of great interest due to injury often occurring in the workplace. In previous papers, UCL reconstruction with autograft has resulted in favorable functional scores and patient satisfaction [1]. In reviewing the articles discussed here, improvement and good to excellent functional scores were reported in all patient cohorts regardless of the score system utilized. The use of allograft versus autograft does not seem to significantly impact functional outcome, but further large-scale studies are needed to identify subtle differences in function.

As UCL injury occurs often in athletes, return to play rates are of particular interest. Previously published studies on UCL reconstruction demonstrate favorable return to play rates. In 2015, Erickson et al. [10] performed a systematic review reporting on return to play rates and functional outcome scores of patients undergoing UCL. Twenty studies were included reviewing 2,019 elbows that underwent UCL reconstruction. They found an overall return to play rate at $86.2 \%$. A study by Marshal etal. [3] evaluated 46 major league baseball pitchers who underwent UCL reconstruction with the use of gracilis or palmaris autograft tendons and did not determine any significant difference in performance outcomes between the two patient cohorts [4]. They found an overall return to play rate of $96 \%$, with $82 \%$ returning to play at the previous MLB level. A larger systematic review and meta-analysis by Peters et al. [15] found an overall return to play of $90 \%$ among all patients who underwent UCL reconstruction. Interestingly, based on level of expertise, 78\% of Major League Baseball players, 67\% of Minor League Baseball Players, 92\% of collegiate players, and $83 \%$ of high school players returned to previous level of play. Erickson et al reported similar levels of return to play as found in these previous studies, and also found that $100 \%$ of patients who underwent UCL reconstruction with allograft returned to play at the same level or higher, although there was no significant difference found due to the high return to play rate in both groups, and the small population size [11]. Savoie et al. [8] also demonstrated a high return to play rate of $83.5 \%$ at or above previous level of play. Overall, there is a high return to play rate utilizing either autograft or allograft for UCL reconstruction, and further studies are needed to demonstrate a significant difference.

As with any surgical procedure, identifying and minimizing postoperative complications is imperative to improving patient outcomes. Complications following UCL reconstruction have been reported in as high as $10.4 \%$ of patients and include in decreasing incidence: ulnar neuritis (77\%), donor site issues (12.5\%), need for revision UCLR (6.6\%), stiffness (2.8\%), reactive synovitis $(1.4 \%)$, post-operative hematoma (0.9\%), ulnar tunnel fracture $(0.9 \%)$ [10]. A meta-regression and systematic review performed by Somerson et al. [16] demonstrated a similar overall complication rate of $10.2 \%$ with the primary complications being ulnar neurapraxia developing in $6.7 \%$ of all patients. Of interest, they found patients were more likely to report excellent or good Conway outcome rating if they did not report an ulnar neurapraxia, demonstrating that neurapraxia is subjectively related to how patients perceive their functional outcome. Erickson et al. [11] found that while there were no differences in complications between the type of graft used, more complications were identified with the standard docking technique that the double-docking technique. Interestingly, allografts were used more often with the double-docking technique, while hamstring autograft were used primarily with the standard docking technique. In Savoie et al, the rate of complications was reported as $6 \%$, though this is a single study and may be the result of a small patient cohort.8 Further studies with equal technique distribution and reporting methods are needed to determine if there is a difference in complication rate and type between allograft and autograft.

The studies identified by our literature search appear to demonstrate comparable functional outcomes, return to play rate, and complication rates with the use of allograft tendon versus autograft tendon in UCL reconstruction. The main limitation of our study was the availability of very few comparative studies directly evaluating autograft versus allograft use in UCL reconstruction. Those that do exist consist of very small patient cohorts which limits the possibility of statistical analysis. While there appear to be trends toward similar, and favorable, outcomes between the two graft options, the heterogeneity of the studies limit data extrapolation. Additionally, the studies reviewed were retrospective in nature, and prospective double-blind control studies are needed for more substantial data. While these limitations exist, there appear to be similar outcomes between graft types, and we feel allograft reconstruction is an appropriate treatment option for UCL reconstruction.

\section{Conclusion}

While there is a paucity of comparative literature regarding the use of allograft versus autograft tendon in UCL reconstruction, there does not appear to be any significant difference in regards to functional scores, return to play rate, or complication rates. Further study into this topic is needed to determine more adequate reliability of our conclusions, although from the available literature it does appear to be an appropriate option while sparing potential complications of donor site morbidity. Overall, allograft reconstruction is an adequate option for ulnar collateral ligament reconstruction.

\section{References}

1. Waris W (1946) Elbow injuries of javelin-throwers. Acta Chir Scand 93(6): 563-575. 
2. Petty DH, Andrews JR, Fleisig GS, Cain EL (2004) Ulnar collatera ligament reconstruction in high school baseball players: clinical results and injury risk factors. Am J Sports Med 32(5): 1158-1164.

3. Marshal N, Keller R, Orr L (2019) Major league baseball pitching performance after Tommy John surgery and the effect of tear characteristics, technique, and graft type. Am J Sports Med 47(3): 713720 .

4. Regan WD, Korinek SL, Morrey BF, An KN (1991) Biomechanical study of ligaments around the elbow joint. Clin Orthop Relat Res (271): 170-179.

5. Molenaars R, Bekerom M, Eygendaal D (2019) The pathoanatomy of the anterior bundle of the medial ulnar collaterallLigament. J Shoulder Elbow Surg 28: 1497-1504.

6. Morrey BF, An KN (1983) Articular and ligamentous contributions to the stability of the elbow joint. Am J Sports Med 11(5): 315-319.

7. Jobe F, Stark H, Lombardo S (1986) Reconstruction of the ulnar collateral ligament in athletes. J Bone Joint Surg Am 68(8): 1158-1163.

8. Savoie F, Morgan C, Yaste J, Hurt J (2013) Medial ulnar collateral ligament reconstruction using hamstring allograft in overhead throwing athletes. J Bone Joint Surg Am 95(12): 1062-1066.

9. Moher D, Liberati A, Tezlaff J, Altman DG (2009) Preferred reporting items for systematic reviews and meta-analyzes: the PRISMA statement. BMJ 339: b2535.
10. Erickson B, Chalmers P, Bush-Joseph C (2015) Ulnar collateral ligament reconstruction of the elbow: A systematic review of the literature. Orthop J Sports Med 3(12): 2325967115618914

11. Erickson B, Cvetanovich G, Frank R (2016) Do clinical results and returnto-sport rates after ulnar collateral ligament reconstruction differ based on graft choice and surgical technique. Orthop J Sports Med 4(11): 2325967116670142

12. Merolla G, Del Sordo S, Paladini P, Porcellini G (2014) Elbow ulnar collateral ligament reconstruction: Clinical, radiographic, and ultrasound outcomes at a mean 3- year follow up. Musculoskelet Surg 98 Suppl 1: 87-93.

13. Hardy A, Casabianca L, Andrieu K (2017) Complications following harvesting of patellar tendon or hamstring tendon grafts for anterior cruciate ligament reconstruction: Systematic review of literature. Orthop Traumatol Surg Res 103(8S): S245-S248.

14. Leslie B, Osterman A, Wolfe S (2017) Inadvertent harvest of the median nerve instead of the palmaris longus tendon. J Bone Joint Surg Am 99(14): 1173-1182.

15. Peters S, Bullock G, Goode A (2018) The success of return to sport after ulnar collateral ligament injury in baseball: A systematic review and meta-analysis. J Shoulder Elbow Surg 27(3): 561-571.

16. Somerson J, Petersen J, Neradilek M (2018) Complications and outcomes after medial ulnar collateral ligament reconstruction: A meta-regression and systematic review. JBJS Rev 6(5): e4.

For possible submissions Click below: 